\title{
Diagnosis and management of non-cystic fibrosis bronchiectasis
}

\author{
Authors: Laura Macfarlane, ${ }^{\mathrm{A}}$ Kartik Kumar, ${ }^{\mathrm{B}}$ Thomas Scoones, ${ }^{\mathrm{C}}$ Andrew Jones, ${ }^{\mathrm{D}}$ Michael R Loebinger ${ }^{\mathrm{E}}$ and Robert Lord ${ }^{\mathrm{F}}$
}

\begin{abstract}
Bronchiectasis is a heterogeneous and increasingly prevalent chronic pulmonary disease that is associated with significant morbidity. In this review, we outline how patients with bronchiectasis may present clinically and describe an approach to its diagnosis, including how to identify an underlying aetiology. We discuss the important considerations when treating either acute exacerbations or stable disease and provide an overview of the role of long-term antimicrobials, airway clearance methods and other supportive management.
\end{abstract}

\section{Introduction}

The term 'bronchiectasis' refers to a heterogeneous group of pulmonary diseases characterised by irreversibly damaged and dilated bronchi. It has a variety of causes and a broad spectrum of clinical presentations, ranging from asymptomatic radiological changes detected incidentally to chronic sputum production and recurrent exacerbations. Bronchiectasis may be considered a syndrome, representing a common endpoint for a number of different disease entities. ${ }^{2}$ From a pathophysiological perspective, airway architectural changes lead to mucus accumulation, chronic airway infection and persistent neutrophilic inflammation. ${ }^{3}$ This causes further airway damage, completing a 'vicious cycle' (Fig 1). ${ }^{3}$ Many conditions may initiate this process, including those related to impaired mucociliary clearance, defects in the immune system and disordered inflammation associated with systemic disease (Table 1).

Authors: ${ }^{\mathrm{A}}$ specialty trainee in respiratory medicine and general internal medicine, Wythenshawe Hospital, Manchester, UK; ${ }^{B}$ specialty trainee in respiratory medicine and general internal medicine and NIHR Imperial BRC clinical research fellow in respiratory medicine, Imperial College London, London, UK and Royal Brompton Hospital, London, UK; ${ }^{C}$ clinical fellow in respiratory medicine, Wythenshawe Hospital, Manchester, UK; ${ }^{D}$ consultant respiratory physician and honorary professor, Wythenshawe Hospital, Manchester, UK and The University of Manchester, Manchester, UK; ${ }^{E}$ consultant physician in respiratory medicine, lead for tuberculosis service, clinical director of laboratory medicine and professor of practice (respiratory medicine), Imperial College London, London, UK and Royal Brompton Hospital, London, UK; ${ }^{F}$ consultant respiratory physician and honorary senior lecturer, Wythenshawe Hospital, Manchester, UK and The University of Manchester, Manchester, UK

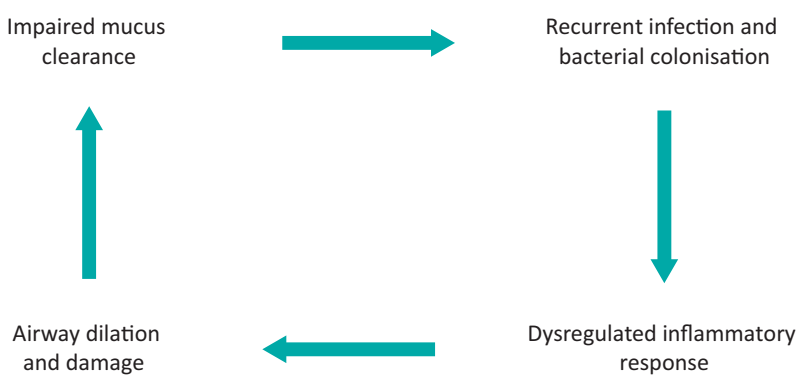

Fig 1. Cole's vicious cycle hypothesis.

\section{Key points}

Patients with a new diagnosis of bronchiectasis should be investigated for the underlying aetiology and referred to a respiratory specialist for further management.

When a patient presents with an acute exacerbation of bronchiectasis, it is important to send sputum for routine microbiological and mycobacterial culture; and to use previous microbiology results to guide prompt antibiotic therapy.

Infection with Pseudomonas aeruginosa can be associated with a worse prognosis and should be managed appropriately; eradication is usually attempted when it is first cultured.

Good airway clearance is paramount in both acute exacerbations and in stable disease; all patients should be reviewed by a physiotherapist, taught airway clearance techniques and provided with a self-management plan.

Long-term antibiotics can be considered by a respiratory specialist if the patient has three or more exacerbations per year.

KEYWORDS: bronchiectasis, Pseudomonas aeruginosa, airway clearance, non-tuberculous mycobacteria, macrolides

DOI: 10.7861/clinmed.2021-0651 


\begin{tabular}{|c|c|}
\hline Cause & Examples \\
\hline \multicolumn{2}{|l|}{ Idiopathic } \\
\hline \multirow[t]{6}{*}{ Post-infective } & Pneumonia \\
\hline & $\begin{array}{l}\text { Tuberculosis (Mycobacterium } \\
\text { tuberculosis) }\end{array}$ \\
\hline & $\begin{array}{l}\text { Non-tuberculous mycobacterial } \\
\text { pulmonary disease }\end{array}$ \\
\hline & Whooping cough (Bordetella pertussis) \\
\hline & Allergic bronchopulmonary aspergillosis \\
\hline & $\begin{array}{l}\text { Respiratory viruses (eg adenovirus and } \\
\text { measles) }\end{array}$ \\
\hline \multirow[t]{2}{*}{ Immunodeficiency } & $\begin{array}{l}\text { Primary (eg common variable immune } \\
\text { deficiency) }\end{array}$ \\
\hline & $\begin{array}{l}\text { Secondary (eg HIV, chemotherapy and } \\
\text { immunosuppressant use) }\end{array}$ \\
\hline \multirow{4}{*}{$\begin{array}{l}\text { Autoimmune } \\
\text { diseases }\end{array}$} & Inflammatory bowel disease \\
\hline & $\begin{array}{l}\text { Connective tissue disease (eg } \\
\text { rheumatoid arthritis, ankylosing } \\
\text { spondylitis and Sjögren's syndrome) }\end{array}$ \\
\hline & Sarcoidosis \\
\hline & Systemic lupus erythematosus \\
\hline \multirow{3}{*}{$\begin{array}{l}\text { Impaired mucociliary } \\
\text { clearance }\end{array}$} & Cystic fibrosis \\
\hline & Primary ciliary dyskinesia \\
\hline & Young's syndrome \\
\hline \multirow{6}{*}{$\begin{array}{l}\text { Airway architectural } \\
\text { distortion, injury or } \\
\text { obstruction }\end{array}$} & Chronic obstructive pulmonary disease \\
\hline & Alpha-1 antitrypsin deficiency \\
\hline & Inhalation of toxins/smoke \\
\hline & $\begin{array}{l}\text { Recurrent aspiration secondary to } \\
\text { dysphagia or gastro-oesophageal reflux }\end{array}$ \\
\hline & Tumour \\
\hline & Foreign body \\
\hline \multirow[t]{3}{*}{ Congenital causes } & Marfan's syndrome \\
\hline & $\begin{array}{l}\text { Mounier-Kuhn syndrome } \\
\text { (tracheobronchomegaly) }\end{array}$ \\
\hline & $\begin{array}{l}\text { Williams-Campbell syndrome (cartilage } \\
\text { deficiency) }\end{array}$ \\
\hline
\end{tabular}

\section{Clinical presentation}

Bronchiectasis is becoming increasingly prevalent, with over 200,000 people estimated to be living with the disease in the UK. ${ }^{4}$ It is more common in women and with increasing age; and it can cause significant morbidity. ${ }^{5}$ The classic symptoms are of chronic cough, excessive sputum production and recurrent respiratory infections. These may be accompanied by pleuritic chest pain, haemoptysis, breathlessness and lethargy. ${ }^{6}$ Bronchiectasis should be suspected in patients with consistent symptoms, particularly in the presence of relevant risk factors, comorbidities or pre-existing respiratory conditions (Table 1). The isolation of pathogenic organisms such as Pseudomonas aeruginosa or non-tuberculous mycobacteria (NTM) in



Fig 2. Computed tomography demonstrating bronchial wall thickening (green arrow), airway larger than the adjacent pulmonary artery branch (yellow arrow) and mucus plugging (blue arrow).

respiratory tract samples, particularly when detected more than once, should also trigger investigations for bronchiectasis. ${ }^{6}$

\section{Diagnosis}

\section{Imaging}

Following a baseline chest X-ray, a high-resolution computed tomography (HRCT) of the chest should be performed to confirm the diagnosis (Fig 2). Bronchiectasis is characterised by the presence of dilated airways, typified by a broncho-arterial ratio $>1$, lack of airway tapering towards the periphery and airways being visible within 1 $\mathrm{cm}$ proximity of the pleura. Non-specific findings (including bronchial wall thickening, tree-in-bud changes and mucus plugging) are frequently present. Mosaic perfusion, a pattern of variable pulmonary attenuation on CT, can be present in small airways diseases including bronchiectasis. It can be differentiated from pulmonary vascular disease, the other main cause of mosaic perfusion, using expiratory images. Persistence of these findings on an expiratory CT indicates airways disease; this radiological finding is termed 'air trapping.?

\section{Establishing aetiology}

An underlying aetiology should be sought using a combination of clinical history, physical examination, thoracic radiology, blood tests and, where appropriate, additional investigations (Table 2 ). ${ }^{8}$ This is important to direct and tailor further management. Despite comprehensive evaluation, many cases of bronchiectasis will be labelled as idiopathic or attributed to a previous severe respiratory tract infection (post-infective). In some patients with consistent histories, diagnoses such as cystic fibrosis and primary ciliary dyskinesia should be considered. Within the appropriate clinical setting, systemic diseases such as inflammatory bowel disease and connective tissue disorders should also be investigated. ${ }^{8}$ 
Table 2. Initial investigations to screen for common causes of bronchiectasis

\begin{tabular}{|c|c|c|c|}
\hline \multirow[b]{2}{*}{ Performed in all patients } & Condition & Clinical features & Investigations \\
\hline & \multirow[t]{4}{*}{ Immunodeficiency } & \multirow[t]{4}{*}{$\begin{array}{l}\text { Recurrent infections (pulmonary } \\
\text { and extra-pulmonary) }\end{array}$} & $\begin{array}{l}\text { Serum immunoglobulins (IgG, } \\
\text { IgA and IgM) }\end{array}$ \\
\hline & & & Serum protein electrophoresis \\
\hline & & & HIV \\
\hline & & & Pneumococcal antibody levels \\
\hline & \multirow{3}{*}{$\begin{array}{l}\text { Allergic bronchopulmonary } \\
\text { aspergillosis (ABPA) }\end{array}$} & \multirow{3}{*}{$\begin{array}{l}\text { Pre-existing diagnosis of } \\
\text { asthma, wheeze, proximal } \\
\text { bronchiectasis, sputum plugs }\end{array}$} & Serum total IgE \\
\hline & & & $\begin{array}{l}\text { Specific IgE and IgG to } \\
\text { Aspergillus fumigatus }\end{array}$ \\
\hline & & & Peripheral blood eosinophils \\
\hline & \multirow{2}{*}{$\begin{array}{l}\text { Bacterial infection / pulmonary } \\
\text { tuberculosis (TB) / non- } \\
\text { tuberculous mycobacterial } \\
\text { (NTM) pulmonary disease }\end{array}$} & \multirow{2}{*}{$\begin{array}{l}\text { Non-specific respiratory } \\
\text { symptoms (cough, sputum } \\
\text { production, haemoptysis, night } \\
\text { sweats, weight loss, pyrexia and } \\
\text { fatigue) }\end{array}$} & Routine sputum culture \\
\hline & & & $\begin{array}{l}\text { Mycobacterial sputum culture } \\
(\times 3)\end{array}$ \\
\hline \multirow{10}{*}{$\begin{array}{l}\text { Performed in patients with } \\
\text { specific clinical phenotypes }\end{array}$} & \multirow[t]{2}{*}{ Cystic fibrosis } & \multirow{2}{*}{$\begin{array}{l}\text { Early onset, family history, } \\
\text { male infertility, malabsorption, } \\
\text { pancreatitis }\end{array}$} & Sodium chloride sweat test \\
\hline & & & CFTR mutation analysis \\
\hline & \multirow[t]{3}{*}{ Primary ciliary dyskinesia } & \multirow{3}{*}{$\begin{array}{l}\text { Neonatal distress, family } \\
\text { history, recurrent otitis media, } \\
\text { rhinosinusitis, infertility and } \\
\text { symptoms since childhood }\end{array}$} & Nasal nitric oxide \\
\hline & & & $\begin{array}{l}\text { Tests assessing ciliary structure } \\
\text { and function }\end{array}$ \\
\hline & & & Genetics \\
\hline & \multirow{4}{*}{$\begin{array}{l}\text { Rheumatoid arthritis, } \\
\text { ankylosing spondylitis, Sjögren's } \\
\text { syndrome and systemic lupus } \\
\text { erythematosus }\end{array}$} & \multirow{4}{*}{$\begin{array}{l}\text { Clinical features consistent } \\
\text { with connective tissue disease, } \\
\text { arthritis or vasculitis }\end{array}$} & Rheumatoid factor \\
\hline & & & Anti-cyclic citrullinated peptide \\
\hline & & & Antinuclear antibodies \\
\hline & & & $\begin{array}{l}\text { Anti-neutrophil cytoplasmic } \\
\text { antibodies }\end{array}$ \\
\hline & Alpha-1 antitrypsin deficiency & Basal pan-acinar emphysema & Alpha-1 antitrypsin \\
\hline
\end{tabular}

\section{Management}

\section{Acute exacerbations}

An acute deterioration in baseline bronchiectasis symptomatology is referred to as an exacerbation. These are typically characterised by increasing cough, dyspnoea and sputum expectoration. The sputum usually, but not always, increases in volume and it becomes more purulent and viscous. There may be haemoptysis and associated systemic symptoms. ${ }^{9}$

Mild exacerbations in the absence of hypoxaemia and haemodynamic instability can usually be managed on an outpatient basis. Prompt courses of oral antibiotics, guided by local antimicrobial policy and the results of previous sputum cultures, are usually required but usually for at least 10-14 days. ${ }^{10}$ Sputum sampling should occur alongside acute antibiotic treatment, as identification of pathogenic species and their antibiotic sensitivities will direct selection of alternative antimicrobials should initial therapy fail.

In more severe exacerbations, intravenous antibiotics for 10-14 days may be used. These can be delivered on an outpatient basis in place of oral therapy if outpatient intravenous antibiotic therapy services are available and provided that the patient is sufficiently stable and adherent to such management. ${ }^{11}$ For example, this may be relevant for a patient who is chronically infected with ciprofloxacin-resistant $P$ aeruginosa or who requires intravenous treatment for an acute exacerbation but is otherwise sufficiently stable to remain at home. Hospital admission for intravenous therapy may be indicated if the exacerbation is severe, the patient would benefit from inpatient physiotherapy or if home management is not practical. It is important to ensure that adequate doses and duration of therapy are used (Box 1).

Acute exacerbations of bronchiectasis may cause mild haemoptysis, which can be managed in the community with oral antibiotics and sometimes tranexamic acid. More significant haemoptysis will, however, necessitate hospital admission. Tranexamic acid, blood products and fluid resuscitation may be required depending on the severity of the haemoptysis and the degree of haemodynamic instability. Bronchial artery embolisation can be performed by an interventional radiologist in some specialist centres. A $0.7 \%-2 \%$ peri-procedural risk of stroke means that careful risk-benefit analysis must be undertaken prior to embolisation and it should only be employed in life-threatening circumstances. ${ }^{12}$ If haemoptysis is massive (for example, more 
Box 1. Practical tips for managing exacerbations of bronchiectasis on the acute medical take
Acute management
> Send sputum for routine culture and mycobacterial culture as soon as possible.
> Review previous sputum cultures and sensitivities to guide antibiotic selection; if unavailable, refer to local microbiology guidelines.
> Modify antibiotic choice based on acute sputum culture result and treat for 10-14 days.
> Optimise airway clearance; liaise with respiratory physiotherapy.
$>$ If significant haemoptysis occurs, consider tranexamic acid.
$>$ Request a review by a respiratory specialist if any concerns.
On discharge
> Refer to respiratory physiotherapy to teach/reinforce good sputum clearance technique.
$>$ Refer to respiratory clinic for follow-up if appropriate.
$>$ Provide patient with sputum pots to send samples to their general practitioner if their symptoms worsen at home.
> Ask general practitioner to provide an antibiotic rescue pack (guided by previous sputum culture results and sensitivities) and provide clear instructions for use.
Offer current smokers a referral to smoking cessation services

than 100-200 mL per 24 hours) and potentially life threatening, the patient should be nursed on their side with the abnormal lung dependent. They may require intubation, ventilation and the consideration of a double lumen endotracheal tube that isolates and occludes the main bronchus supplying the bleeding lung while allowing the patient to be ventilated via the normal lung.

\section{Airway clearance}

Effective airway clearance, coupled with maintaining good hydration, is an integral component of managing bronchiectasis, both in the long term and in the context of an acute exacerbation. All patients should be encouraged to engage with physiotherapy services. Unfortunately, not all hospitals have a dedicated respiratory physiotherapist service. Airway clearance techniques can, however, be taught by all physiotherapists and patients can be referred to a specialist chest physiotherapist if symptoms persist. They should be provided with an individualised airway clearance plan, which can aid sputum expectoration, reduce cough frequency and decrease dyspnoea. ${ }^{7}$ Patients should escalate their individualised airway management regimens during an exacerbation. In acute exacerbations necessitating hospitalisation, inpatient physiotherapy review should be sought to optimise airway clearance.

Mucolytics can be used as an adjunct to chest clearance. Hypertonic saline $(6 \%-7 \%)$ is used as a first-line treatment. An observed test dose is required due to the risk of bronchoconstriction. ${ }^{13}$ Although commonly prescribed, there is little evidence to support the use of carbocisteine. If used, it should be trialled for 6 months and only continued if it is effective at improving airway clearance. Carbocisteine is contraindicated if there is a history of active peptic ulceration. Nebulised recombinant deoxyribonuclease should not be used outside of cystic fibrosis.?

\section{Vignette 1}

Presentation

A 60-year-old man presented to ambulatory care with a worsening cough productive of purulent sputum and shortness of breath. His general practitioner (GP) started doxycycline 3 days previously, but his symptoms continued to worsen. He reported producing half an eggcup full of yellow-green phlegm each day and suffering from chest infections every winter. A sputum culture recently sent by his GP had grown Pseudomonas aeruginosa. Observations were within normal limits. Clinical examination revealed coarse inspiratory crepitations at the lung bases.

Action

Following a baseline chest X-ray, a high-resolution computed tomography of the chest showed severe bi-basal bronchiectasis. He was treated with a 2-week course of oral ciprofloxacin having been warned about the risk of drug interactions and Achilles tendonitis. He was referred to the respiratory clinic for further investigation of his bronchiectasis.

\section{Outcome}

Repeat sputum cultures grew normal respiratory tract flora, suggesting eradication of $P$ aeruginosa. Investigations for an underlying aetiology for the bronchiectasis were normal. He was referred to the respiratory physiotherapists and taught airway clearance techniques, which he uses daily. He subsequently reported an improvement in his cough and breathlessness. He was offered the pneumococcal vaccine and annual influenza vaccination.

\section{First growth of $P$ aeruginosa}

Due to poor outcomes associated with $P$ aeruginosa, eradication is usually attempted when it is first cultured in respiratory samples using high-dose oral ciprofloxacin for 14 days. A repeat sputum sample should then be sent. If $P$ aeruginosa isolation persists, a combination of intravenous and nebulised antipseudomonal antibiotics can be considered. ${ }^{7}$ Patients are considered colonised if they continue to isolate $P$ aeruginosa despite eradication therapy (Vignettes 1 and 2).

\section{Long-term antibiotics}

If patients have three or more exacerbations per year despite optimal airway clearance, and prompt treatment and exclusion of other treatable traits, long-term antibiotics should be considered. The choice of long-term antibiotic is determined by the patient's colonisation status.

In patients who are not colonised by $P$ aeruginosa, long-term macrolides are offered as a first-line therapy. Caution is needed when initiating these due to the risk of QT interval prolongation and hearing impairment. Additionally, as macrolides form the mainstay of NTM treatment, active NTM infection should be excluded by sputum mycobacterial culture prior to long-term macrolide initiation. Long-term antibiotics should be started after careful risk-benefit analysis by a respiratory specialist to ensure judicious antimicrobial use. ${ }^{14}$ In chronic $P$ aeruginosa colonisation, nebulised antibiotics (such as colomycin) are currently used as a 


\section{Vignette 2}

Presentation

A 55-year-old woman with a history of post-infective

bronchiectasis was admitted to the acute medical unit with a fever, hypoxaemia, worsening breathlessness and a cough productive of mucopurulent sputum. She had experienced two similar exacerbations recently. Six months previously, sputum cultures grew Streptococcus pneumoniae and she recovered following a 2-week course of amoxicillin. Six weeks previously, she had a further exacerbation, with sputum cultures growing Pseudomonas aeruginosa for the first time. She initially responded well to a 2 -week course of oral ciprofloxacin. She reported performing airway clearance exercises on a daily basis.

Action

A chest X-ray at admission showed consolidation. Sputum cultures on this admission confirmed growth of a fully sensitive $P$ aeruginosa for a second time. As she failed to eradicate $P$ aeruginosa and she had not had a sustained response to ciprofloxacin, she was started on intravenous piperacillintazobactam for an extended course. She received regular chest physiotherapy during her admission.

Outcome

She responded well to parenteral therapy. When no longer requiring oxygen, she was discharged to complete a 2-week course of intravenous antibiotics at home. To complete eradication therapy, she was offered 3 months of nebulised colomycin

first-line treatment to reduce exacerbation frequency, with longterm oral macrolides as an alternative. ${ }^{7,15}$

If patients continue to have frequent exacerbations despite these interventions, a combination of nebulised and oral antibiotics can be helpful; and if this fails, cyclical intravenous antibiotics can be initiated on a case-by-case basis. ${ }^{7}$

\section{Additional supportive management}

All patients with bronchiectasis should be offered the pneumococcal vaccine, annual influenza vaccination, COVID-19 vaccination and, where relevant, smoking cessation advice. Maintenance of a healthy body weight and physical fitness should be encouraged through dietary advice and exercise. A low body mass index in bronchiectasis patients is associated with poor outcomes. ${ }^{16}$ Dietitian input should be sought if appropriate. Breathless patients should be encouraged to attend pulmonary rehabilitation and offered a trial of inhaled bronchodilators if the dyspnoea is resulting in significant limitation. ${ }^{7,17}$ There is no role for routine administration of inhaled or oral corticosteroids, although trials of inhaled corticosteroids may be appropriate for patients with inflammatory bowel disease or coexistent asthma, whereas oral steroids and/or antifungal agents are used for allergic bronchopulmonary aspergillosis.?

\section{Associated conditions}

Non-tuberculous mycobacterial pulmonary disease NTM are ubiquitous environmental organisms frequently found in water and soil. Pulmonary NTM infections increase
Box 2. Diagnostic criteria for non-tuberculous

\section{mycobacterial pulmonary disease}

Clinical, microbiological and radiological criteria must each be fulfilled.

\section{Clinical}

$>$ History of pulmonary symptoms in keeping with nontuberculous mycobacterial pulmonary disease.

$>$ Other possible diagnoses should have been excluded.

\section{Radiological}

> High-resolution computed tomography of the chest showing multifocal bronchiectasis with multiple small nodules.

\section{Microbiological}

$>$ At least two sputum cultures positive for non-tuberculous mycobacteria.

> OR one bronchial wash or bronchial lavage culture positive for non-tuberculous mycobacteria.

$>$ OR a lung biopsy with histology consistent with nontuberculous mycobacteria plus a biopsy or sputum result positive for non-tuberculous mycobacteria.

the risk of bronchiectasis developing and bronchiectasis itself predisposes patients to NTM lung disease. Mycobacterium avium complex is the most commonly isolated NTM in patients with bronchiectasis. ${ }^{18}$ A diagnosis of NTM pulmonary disease requires clinical, microbiological and radiological criteria to be fulfilled (Box 2). ${ }^{19}$ A minimum of three antimicrobials are required; depending on the NTM species and response to treatment, parenteral and/or inhaled antimicrobial treatment may be indicated. Cases should be managed in conjunction with or by specialists who have experience in treating NTM pulmonary disease.

\section{Allergic bronchopulmonary aspergillosis}

Allergic bronchopulmonary aspergillosis (ABPA) is a condition that complicates asthma and cystic fibrosis, and is caused by hypersensitivity to the environmental mould Aspergillus fumigatus. It causes bronchiectasis that is typically central and may be present at diagnosis or develop as the disease progresses. ABPA often presents with symptoms of worsening asthma control. Acute exacerbations can cause fever, haemoptysis, expectoration of sputum plugs and pulmonary infiltrates on imaging. Diagnosis of ABPA is based on demonstration of hypersensitivity to $A$ fumigatus (using skin-prick tests or specific immunoglobulin $E(\operatorname{IgE})$ ), raised total IgE and peripheral blood eosinophilia. An aim of ABPA treatment is to prevent the development or progression of bronchiectasis. Treatment is with a tapering course of steroids in the first instance. Antifungals may be required if steroids fail to control symptoms. ${ }^{20}$ Pulmonary aspergillomas, another manifestation of Aspergillus lung disease in which a fungal ball forms in areas of damaged lung, can also occur in patients with bronchiectasis, they may cause haemoptysis, which can be massive. ${ }^{21}$ Depending on the severity and associated symptoms, treatment of pulmonary aspergillomas may entail systemic and/ or intracavitary instillation of antifungals, and surgical resection of the affected lung region. 


\section{Vignette 3}

Presentation

A 35 -year-old man with a body mass index of $19 \mathrm{~kg} / \mathrm{m}^{2}$ was admitted to hospital under the surgical team with acute pancreatitis. He had no known past medical history but had recently visited his general practitioner due to difficulties conceiving with his partner. During the inpatient admission, he developed a worsening cough with green sputum production and occasional small-volume haemoptysis. He developed an oxygen requirement of $1 \mathrm{~L} / \mathrm{min}$. He was initially treated with antibiotics as per local protocol for a presumed hospital-acquired pneumonia pending sputum culture results.

Action

A high-resolution computed tomography of the chest showed bilateral bronchiectasis with upper lobe predominance. He was reviewed by the respiratory physiotherapists for airway clearance techniques but initially struggled to clear large volumes of viscous sputum, so he was started on regular nebulised hypertonic saline after an observed test dose. Sputum cultures subsequently grew methicillin-sensitive Staphylococcus aureus. Sputum mycobacterial cultures were negative. His antibiotic was changed to flucloxacillin. Serum immunoglobulins and serum electrophoresis were normal. An HIV test was negative.

Outcome

After a few days of parenteral flucloxacillin and regular chest physiotherapy, his symptoms improved and his oxygen requirement resolved. He was switched to oral flucloxacillin and provided with an airway clearance self-management plan prior to discharge. He was referred to the respiratory clinic for further investigation. In view of the history of pancreatitis, possible infertility and suspected malabsorption underlying his low body mass index, sodium chloride sweat testing and cystic fibrosis transmembrane conductance regulator mutation analysis were arranged to investigate for cystic fibrosis.

\section{Follow-up}

Not all patients with bronchiectasis require follow-up routinely in secondary care. Referral is appropriate to confirm the diagnosis and investigate underlying causes. Patients may be managed in primary care if they do not exacerbate frequently, but primary care colleagues may need specific advice about antibiotic selection, dose and course duration. More complex patients will, however, need follow-up in specialist clinics. This includes patients who are clinically deteriorating; are experiencing three or more exacerbations per year; are on long-term antibiotics; have a systemic disease underlying their bronchiectasis; or are chronically colonised with certain pathogens ( $P$ aeruginosa, NTM or methicillin-resistant Staphylococcus aureus; Vignette 3). Referral to an immunology clinic may be appropriate if an underlying immunodeficiency is suspected.

\section{Conclusion}

Bronchiectasis is a common chronic respiratory condition. The diagnosis is based on the presence of classic symptoms and confirmed by supportive radiology. First-line therapy is airway clearance. In patients whose symptoms are not improving, mucolytics or long-term antibiotics should be considered. Pulmonary exacerbations should be treated promptly with appropriate antibiotics by the appropriate route and at the correct dose and course length. Wherever possible the aetiology should be established in order to tailor the management plan to individual patient needs.

\section{Conflicts of interest}

Michael R Loebinger declares honoraria from Grifols, Insmed, AstraZeneca, Savara and Armata. Robert Lord reports an independent grant from Vertex Pharmaceuticals for an investigator-initiated study on gastro-oesophageal reflux.

\section{References}

1 José RJ, Loebinger MR. Clinical and radiological phenotypes and endotypes. Semin Respir Crit Care Med 2021;42:549-55.

2 Flume PA, Chalmers JD, Olivier KN. Advances in bronchiectasis: endotyping, genetics, microbiome, and disease heterogeneity. Lancet 2018:392:880-90.

3 McShane PJ, Naureckas ET, Tino G, Strek ME. Non-cystic fibrosis bronchiectasis. Am J Respir Crit Care Med 2013;188:647-56.

4 Snell N, Gibson J, Jarrold I, Quint JK. Epidemiology of bronchiectasis in the UK: Findings from the British Lung Foundation's 'Respiratory health of the nation' project. Respir Med 2019;158:21-3.

5 Quint JK, Millett ER, Joshi M et al. Changes in the incidence, prevalence and mortality of bronchiectasis in the UK from 2004 to 2013: a population-based cohort study. Eur Respir J 2016;47:186-93.

6 Chalmers JD, Aliberti S, Blasi F. Management of bronchiectasis in adults. Eur Respir ] 2015;45:1446-62.

7 Hill AT, Sullivan AL, Chalmers JD et al. British Thoracic Society Guideline for bronchiectasis in adults. Thorax 2019;74(Suppl 1): $1-69$.

8 Pasteur MC, Helliwell SM, Houghton SJ et al. An investigation into causative factors in patients with bronchiectasis. Am J Respir Crit Care Med 2000;162(4 Pt 1):1277-84

9 Hill AT, Haworth CS, Aliberti S et al. Pulmonary exacerbation in adults with bronchiectasis: a consensus definition for clinical research. Eur Respir ] 2017:49:1700051.

10 National Institute for Health and Care Excellence. Bronchiectasis (non-cystic fibrosis), acute exacerbation: antimicrobial prescribing: NICE guideline [NG117]. NICE, 2018.

11 López-Cortés LE, Ayerbe-García R, Carrasco-Hernández L et al Outpatient parenteral antimicrobial treatment for non-cystic fibrosis bronchiectasis exacerbations: a prospective multicentre observational cohort study. Respiration 2019;98:294-300.

12 Panda A, Bhalla AS, Goyal A. Bronchial artery embolization in hemoptysis: a systematic review. Diagn Interv Radiol 2017;23:307-17.

13 O'Neill K, O'Donnell AE, Bradley JM. Airway clearance, mucoactive therapies and pulmonary rehabilitation in bronchiectasis. Respirology 2019;24:227-37.

14 Serisier DJ. Risks of population antimicrobial resistance associated with chronic macrolide use for inflammatory airway diseases. Lancet Respir Med 2013;1:262-74.

15 Chalmers JD, Boersma W, Lonergan $\mathrm{M}$ et al. Long-term macrolide antibiotics for the treatment of bronchiectasis in adults: an individual participant data meta-analysis. Lancet Respir Med 2019:7:845-54.

16 Qi Q, Li T, Li JC, Li Y. Association of body mass index with disease severity and prognosis in patients with non-cystic fibrosis bronchiectasis. Braz J Med Biol Res 2015:48:715-24.

17 Lee AL, Hill C], Cecins N et al. The short and long term effects of exercise training in non-cystic fibrosis bronchiectasis-a randomised controlled trial. Respir Res 2014;15:44. 
18 Wickremasinghe M, Ozerovitch LJ, Davies G et al. Nontuberculous mycobacteria in patients with bronchiectasis. Thorax 2005;60:1045-51.

19 Griffith DE, Aksamit T, Brown-Elliott BA et al. An official ATS/IDSA statement: diagnosis, treatment, and prevention of nontuberculous mycobacterial diseases. Am J Respir Crit Care Med 2007;175:367416.

20 Kosmidis C, Denning DW. The clinical spectrum of pulmonary aspergillosis. Thorax 2015;70:270-7.
21 De Soyza A, Aliberti S. Bronchiectasis and Aspergillus: How are they linked? Medical Mycology 2017;55:69-81.

Address for correspondence: Dr Laura Macfarlane, North West Lung Centre, Wythenshawe Hospital, Southmoor Road, Wythenshawe, Manchester M23 9LT, UK.

Email: laura.macfarlane@mft.nhs.uk 